\title{
Introduction: Complementarity in Research Syntheses
}

\section{Rod Ellis}

University of Auckland and Shanghai International Studies University

This special issue of Applied Linguistics has two primary goals. The first is to examine the complementarity of two ways of conducting a research synthesis - by means of traditional narrative reviews and by meta-analysis. The second is to accumulate information on three separate topics all related to the role that instruction plays in second language learning. These topics were (1) the role of language aptitude, (2) the effectiveness of $L 2$ pronunciation instruction, and (3) Processing Instruction. It is anticipated therefore that the contents of the special issue will be of interest to both researchers interested in the methodology of conducting research syntheses and those interested in the role of instruction. In this introduction, however, I will focus only on the issue of complementarity of the two ways of conducting a research synthesis.

\section{Some definitions}

Research synthesis serves as a cover term for any attempt to review the literature related to a single topic, which can be either narrowly or broadly defined. Of the topics addressed in the six articles two of them were relatively broadly defined (language aptitude and L2 pronunciation instruction) and one was much more narrowly defined (Processing Instruction). In the context of this special issue, complementarity refers to ways in which two different approaches to conducting a research synthesis can in combination provide a more complete, unified explanation of a phenomenon than either single approach. In other words, if want to know what research has told us about the role of instruction in language learning we benefit most if we combine a narrative and a meta-analytic synthesis of the research.

A narrative review aims to "scope out and 'tell a story' about the empirical territory in order to identify a gap in which to situate new primary research as is customary in the literature review sections of dissertations and empirical articles" (Norris and Ortega, 2006: 5). Norris and Ortega go on to mention and birefly define a number of other types of reviews - the athoritative tour, the comprehensive bilbiographical review, the vote-counting review and the historiographical review, the integrative review and the critical review. However, the distinctions between these types of review are not (and probably cannot) be clearly specified. What they have in common is a qualitative approach where particular primary studies are selected for analysis, described, and evaluated with a view to reaching a number of generalizations about the topic under review. It is this commonality that we wish to capture by the label 'narrative review'.

It is sometimes claimed that a narrative review is not systematic as in this quotation from the introduction to an article on research reviews in psychiatry:

Narrative reviews tend to be mainly descriptive, do not involve a systematic search of the literature, and thereby often focus on a subset of studies in an area chosen based on availability or author selection. Thus narrative reviews while informative can often include an element of selection bias. They can also be confusing at times, particularly if similar studies have diverging results and conclusions. (Uman 2011; 59). 
However, while this may the case with some narrative reviews it is not an essential feature of this type of review. A narrative review can be systematic. To encourage this, the narrative reviews in this special issue drew on the same set of primary studies as the meta-analytic reviews. Also, a good narrative review should aim to discuss and explain diverging results as is the case in all the narrative reviews.

A meta-analysis differs from a narrative review in one key characteristic - it makes use of quantitative procedures to statistically combine the results of studies. Key characteristics of a metaanalysis are that it makes explicit the basis on which primary studies were selected and that it focuses on the analysis of the data contained in these studies rather than on the interpretations and conclusions of the primary researchers. The methodology of a meta-analysis is closely alligned with that of primary study. The research topic is defined, research questions are developed, data is collected (i.e. the results reported in the primary studies), the method used to analyze the data is made explicit, results are reported and discussed and some conclusions reached.

References

Uman, L. 2011. Systematic reviews and meta-analyses. J Can Acad Child Adolesc Psychiatry. Feb 2011; 20(1): 57-59. 